\title{
THE STONE-WEIERSTRASS THEOREM FOR WALLMAN RINGS
}

\author{
H. L. BENTLEY and B. J. TAYLOR
}

(Received 20 May 1976)

Communicated by E. Strzelecki

\begin{abstract}
Biles has called a subring $A$ of the ring $C(X)$ a Wallman ring on $X$ whenever $Z(A)$, the zero sets of function belonging to $A$, forms a normal base on $X$ in the sense of Frink (1964). In the following, we are concerned with the uniform topology of $C(X)$. We formulate and prove some generalizations of the Stone-Weierstrass theorem in this setting.
\end{abstract}

Subject classification (Amer. Math. Soc. (MOS) 1970): 54 C 30, 54 C 40, 54 C 50.

\section{Introduction}

Wallman (1938) gave a method for associating a compact $T_{1}$ space $w(F)$ with a distributive lattice $F ; w(F)$ is the space of all $F$-ultrafilters and the topology of $w(F)$ has as a base for closed sets a lattice $F^{*}$ which is isomorphic to the lattice $F$. Frink (1964) defined the concept of a normal base $F$ on a Tychonoff space $X$ and he applied Wallman's construction to obtain Hausdorff compactifications $w(F)$ of $X$. Throughout this paper, $X$ will denote a Tychonoff space (= completely regular + Hausdorff).

1.1. Definition. A collection $F$ of closed subsets of $X$ is called a lattice of closed subsets of $X$ provided that:

(1) $\varnothing, X \in F$; and

(2) if $A, B \in F$ then $A \cap B \in F$ and $A \cup B \in F$.

1.2. Definition. A base $F$ for the closed subsets of $X$ is called a normal base on $X$ provided :

(1) $F$ is a lattice of closed subsets of $X$.

(2) $F$ is disjunctive (that is, if $A \in F$ and $x \in X-A$, then there exists $B \in F$ with $x \in B$ and $A \cap B=\phi$ ).

(3) $F$ is normal (that is, if $A, B \in F$ with $A \cap B=\varnothing$, then there exist $C, D \in F$ with $A \cap D=\varnothing, B \cap C=\varnothing$ and $C \cup D=X$ ).

If $F$ is a normal base on $X$, then $w(F)$ is the set of all $F$-ultrafilters which becomes a space as follows: If $A \in F$, let $A^{*}$ be the set of all $F$-ultrafilters having $F$ as a member. $F^{*}$ then denotes the set of all $A^{*}$ with $A \in F . F^{*}$ is a base for the closed 
sets of a topology on $w(F) . w(F)$ with this topology is always a Hausdorff compactification of $X$. Here $X$ is embedded into $w(F)$ by the map which sends each point $x \in X$ to the $F$-ultrafilter $\{A \in F \mid x \in A\}$.

Frink observed that the family $Z(X)$ of all zero sets of continuous real valued functions on $X$ is a normal base on $X$ which gives rise to a compactification $w(Z(X))$ equivalent to the Stone-Cech compactification $\beta X$ of $X$. He also observed that if $Y$ is any given compactification (all spaces are Hausdorff) of $X$, and if $E(X, Y)$ denotes the subset of $C(X)$ consisting of those real-valued continuous functions on $X$ which are continuously extendible to all of $Y$, then $Z(E(X, Y))$, the zero sets of such functions, is a normal base on $X$. Biles (1970) later called a subring $A$ of $C(X)$ a Wallman ring on $X$ provided $Z(A)$, the zero sets of functions in $A$, is a normal base on $X$. Bentley and Taylor (1975) studied relationships between algebraic properties of a Wallman ring $A$ and topological properties of the cômpactification $w(Z(A))$ of $X$.

We adopt our notation and terminology from our two earlier papers; these are mostly consistent with that of Gillman and Jerison (1960).

\section{Generalizations of the Stone-Weierstrass Theorem}

We investigate the consequences of having a Wallman ring which is uniformly closed; that is, closed in the uniform topology of $C(X)$. Two theorems motivate this work. One is Urysohn's Extension Theorem which states: "A subspace $S$ of $X$ is $C^{*}$-embedded in $X$ if and only if any two completely separated sets in $S$ are completely separated in $X$." The proof of this theorem as it appears in Gillman and Jerison uses the uniform closeness of $C^{*}(X)$ to construct a function in $C^{*}(X)$ whose restriction to $S$ is a given function in $C^{*}(S)$. The other is the StoneWeierstrass Theorem for real-valued functions which states: "If $Y$ is compact and $A$ is a closed subalgebra of $C(Y)$ which separates points and contains a nonzero constant function then $A=C(Y)$."

In generalizing the Stone-Weierstrass Theorem, we will consider a compactification $Y$ of a space $X$ and a Wallman ring $A$ on $X$ which is a closed subalgebra of $E(X, Y)$. This means each function $f \in A$ is extendible to $Y$. Therefore in much of what follows our Wallman rings will satisfy certain extendibility hypotheses.

We start by presenting a condition which implies that a Wallman ring $A$ contains only functions which are extendible to $w(Z(A))$.

2.1. Definition (Isbell, 1958). $A \subset C(X)$ is closed under composition if and only if for-each $f \in A$ and $g \in C(R), g \circ f \in A$.

2.2. TheOREM. Let $A \subset C(X)$ be closed under composition, then $Z(A)=\left\{f^{-1}[B]: B\right.$ is closed in $R$ and $f \in A\}$. 
Proof. Let $B$ be closed in $R . B$ is a zero set of $C(R)$ so there is a function $g \in C(R)$ such that $B=Z(g)$. Let $f \in A$, then $f^{-1}[B]=Z(g \circ f) \in Z[A]$. Conversely if $F$ is a zero set of $A, F=Z(f)$ for some $f \in A$ and $F=f^{-1}[\{0\}]$.

We will need to use the Taimanov Theorem.

Talmanov Theorem (Taĭmanov, 1952). Let $X$ be dense in $Y$ and let $f: X \rightarrow T$ be a continuous map of $X$ into a compact space $T$. Then $f$ can be extended over $Y$ if and only if for any two subsets $B_{1}$ and $B_{2}$ which are closed in $T$ and disjoint, we have $\mathrm{Cl}_{Y}\left(f^{-1}\left[B_{1}\right]\right) \cap \mathrm{Cl}_{Y}\left(f^{-1}\left[B_{2}\right]\right)=\varnothing$.

2.3. THEOREM. Let $A$ be a Wallman ring on $X$ such that $A$ is closed under composition and $A \subset C^{*}(X)$, then $A \subset E(X, w(Z(A)))$.

Proof. Let $f \in A$ and let $F$ be a compact subset of $R$ such that $f(X] \subset F$. Let $B_{1}$ and $B_{2}$ be disjoint closed subsets of $F$. Then $f^{-1}\left[B_{1}\right]$ and $f^{-1}\left[B_{2}\right]$ are disjoint zero sets of $A$ and

$$
\mathrm{Cl}_{w(Z(A))} f^{-1}\left[B_{1}\right] \cap \mathrm{Cl}_{w(Z(A))} f^{-1}\left[B_{2}\right]=\varnothing .
$$

Therefore, by the Taĭmanov Theorem, $f$ has an extension to $w(Z[A])$.

To further our investigation we make the following definitions which generalize the "completely separated" concept from Urysohn's Extension Theorem.

2.4. Definition. Let $F$ be a family of subsets of $X$ and let $L \subset C(X)$. Then $L$ discriminates $F$-sets if and only if $F_{1}, F_{2} \in F, F_{1} \cap F_{2}=\varnothing$ and $a, b \in R$ implies there is a function $f \in L$ such that $f\left[F_{1}\right] \subset\{a\}$ and $f\left[F_{2}\right] \subset\{b\}$.

2.5. Definition. If $L \subset C(X)$, then

(1) $L$ discriminates points of $X$ if and only if $L$ discriminates $\{\{x\}: x \in X\}-$ sets;

(2) $L$ discriminates compact sets of $X$ if and only if $L$ discriminates

$\{K \subset X: K$ is compact $\}$-sets.

2.6. THEOREM. Let $L$ be a sublattice of $C(X)$ which contains the real constants. If $L$ discrimiantes points of $X$, then $L$ discriminates compact sets of $X$.

Proof. Let $F_{1}$ and $F_{2}$ be disjoint compact subsets of $X$ and let $a, b \in R$. If $a=b$, then the constant function $f=a$ yields $f\left[F_{1}\right]=\{a\}$ and $f\left[F_{2}\right]=\{b\}$. Suppose $a \neq b$. Let $b>a$ and set $\varepsilon=b-a$. For each $x \in F_{1}, y \in F_{2}$ there is a function $f_{x y} \in L$ such that $f_{x y}(x)=a-\varepsilon$ and $f_{x y}(y)=b+\varepsilon$.

Let $G_{x y}=\left\{z \in X: f_{x y}(z)<a\right\}$. Then $x \in G_{x y}$ and so $F_{1} \subset \bigcup_{x_{\in} F_{1}} G_{x y}$. Since $F_{1}$ is compact, there exist $x_{1}, \ldots, x_{n} \in F_{1}$ such that $F_{1} \subset \bigcup_{i=1}^{n} G_{x_{i} y}$. Let

$$
g_{y}=\left(\inf \left\{f_{x_{i} y}: i=1, \ldots, n\right\}\right) \vee a \text {. }
$$


If $z \in F_{1}$ then $z \in G_{x_{i} y}$ for some $i \in\{1, \ldots, n\}$ and $f_{x_{i} y}(z)<a$ which implies $g_{y}(z)=a$. Therefore $g_{y}\left[F_{1}\right] \subset\{a\}$.

Let $H_{y}=\left\{z \in H: g_{y}(z)>b\right\} . f_{x_{i} y}(y)=b+\varepsilon$ for $i=1, \ldots, n$, and so

$$
\left(\inf \left\{f_{x_{i} y}: i=1, \ldots, n\right\}\right)(y)>b \text { and } g_{y}(y)>b .
$$

Therefore $y \in H_{y}$.

Now we let $y$ vary. $F_{2} \subset \bigcup_{y \in F_{2}} H_{y}$. Since $F_{2}$ is compact, there are $y_{1}, \ldots, y_{m} \in F_{2}$ such that $F_{2} \subset \bigcup_{j=1}^{m} H_{y_{j}}$. Let $h=\left(\sup \left\{g_{y_{j}}: j=1, \ldots, m\right\}\right) \wedge b$. If $z \in F_{1}$ then $g_{y}(z)=a$ for each $y \in F_{2}$ and $\left(\sup \left\{g_{y_{j}}: j=1, \ldots, m\right\}\right)(z)=a$ which implies $h(z)=a$. Therefore $h\left[F_{1}\right] \subset\{a\}$. If $z \in F_{2}$ then there exists $k \in\{1, \ldots, m\}$ such that $z \in H_{y_{k}}$ and so $g_{y_{k}}(z)>b$ which implies that $\left(\sup \left\{g_{y_{j}}: j=1, \ldots, m\right\}\right)(z)>b$ and finally that $h(z)=b$. Therefore $h\left[F_{2}\right] \subset\{b\} . h \in L$ since $L$ is a lattice and $L$ contains the constant functions.

2.7. THEOREM. If $F$ is a normal base on $X$, then $E(X, w(F))$ is a sublattice of $C(X)$ which contains all real constants.

Proof. If $f, g \in E(X, w(F))$, then there are $f^{\prime}$ and $g^{\prime} \in C(w(f))$ such that $f=f^{\prime} \mid X$ and $g=g^{\prime} \mid X . f^{\prime} \wedge g^{\prime}$ and $f^{\prime} \vee g^{\prime} \in C(w(F))$ so $f \wedge g=\left(f^{\prime} \wedge g^{\prime}\right) \mid X \in E(X, w(F))$ and $f \vee g=\left(f^{\prime} \vee g^{\prime}\right) \mid X \in E(X, w(F))$. Therefore $E(X, w(F))$ is a sublattice of $\left.C(X)\right)$. Obviously the real constants are in $E(X, w(F))$.

Since $E(X, w(F))$ is a lattice, we can consider sublattices of $E(X, w(F))$. We find that a sublattice of $E(X, w(F))$ which contains the real constants discriminates $F$-sets if and only if the extensions of functions from this sublattice discriminate points of $w(F)$.

2.8. THEOREM. If $F$ is a normal base on $X, L$ is a sublattice of $E(X, w(F))$ which contains the real constants, and $H=\{f \in C(w(F)): f \mid X \in L\}$, then $H$ discriminates points of $w(F)$ if and only if $L$ discriminates $F$-sets.

Proof. Assume $H$ discriminates points of $X$. Let $F_{1}$ and $F_{2} \in F$ such that $F_{1} \cap F_{2}=\varnothing$, and let $a, b \in R . \mathrm{Cl}_{w(F)} F_{1}$ and $\mathrm{Cl}_{w(F)} F_{2}$ are disjoint, compact subsets of $w(F)$. By Theorem 2.6, $H$ discriminates compact sets of $w(F)$, so there exists a function $g \in H$ such that

$$
(g \mid X)\left[F_{1}\right] \subset g\left[\mathrm{Cl}_{w(F)} F_{1}\right] \subset\{a\} \text { and }(g \mid X)\left[F_{2}\right] \subset g\left[\mathrm{Cl}_{w(F)} F_{2}\right] \subset\{b\} .
$$

$g \mid X \in L$ and so $L$ discriminates $F$-sets.

Now assume $L$ discriminates $F$-sets. Let $x, y \in w(F)$ such that $x \neq y$ and let $a, b \in R$. There exist $F_{1}$ and $F_{2}$ in $F$ such that $x \in \mathrm{Cl}_{w(F)} F_{1}, y \in \mathrm{Cl}_{w(F)} F_{2}$ and $F_{1} \cap F_{2}=\varnothing$. Then there exists $f \in L$ such that $f\left[F_{1}\right] \subset\{a\}$ and $f\left[F_{2}\right] \subset\{b\}$. Also, there is a function $g \in H$ such that $g \mid X=f$. Then $g(x) \in \mathrm{Cl}_{w(F)}\left(f\left[F_{1}\right]\right) \subset\{a\}$ and $g(y) \in \mathrm{Cl}_{w(F)}\left(f\left[F_{2}\right]\right) \subset\{b\}$.

We are interested in subsets of $C(X)$ which discriminate their own zero sets so we make the following definition. 
2.9. Definition. Let $A$ be a subset of $C(X)$, then $A$ is discriminating if and only if the following condition is satisfied: $F_{1}, F_{2} \in Z(A), F_{1} \cap F_{2}=\varnothing$ and $a, b \in R$ implies there is a function $f \in A$ such that $f\left[F_{1}\right] \subset\{a\}$ and $f\left[F_{2}\right] \subset\{g\}$.

2.10. TheOREM. Let $A$ be a subset of $C(X)$, then $A$ is discriminating if and only if $A$ discriminates $Z(A)$-sets.

2.11. THEOREM. If $A$ is an inverse closed Wallman ring on $X$ which contains all the real constants, then $A$ is discriminating.

Proof. Let $F_{1}$ and $F_{2} \in Z(A)$ such that $F_{1} \cap F_{2}=\emptyset$, and let $a, b \in R$. There are functions $f_{1}$ and $f_{2} \in A$ such that $F_{1}=Z\left(f_{1}\right)$ and $F_{2}=Z\left(f_{2}\right)$. Let

$$
g=(b-a)\left[f_{1}^{2} /\left(f_{1}^{2}+f_{2}^{2}\right)\right]+a .
$$

Then $g \in A, g\left[F_{1}\right] \subset\{a\}$ and $g\left[F_{2}\right] \subset\{b\}$.

If we consider what happens when $E(X, Y)$ is discrimintaing we obtain the following theorem.

2.12. THEOREM. Let $Y$ be a compactification of $X$, then $Y \cong w(Z[E(X, Y)])$ if and only if $E(X, Y)$ is discriminating.

Proof. Assume $Y \cong w(Z(E(X, Y)))$. Let $H_{1}, H_{2} \in Z(E(X, Y))$ such that $H_{1} \cap H_{2}=\varnothing$, and let $a, b \in R$. $Y$ is a normal space and $\mathrm{Cl}_{Y} H_{1} \cap \mathrm{Cl}_{Y} H_{2}=\varnothing$, so there is a function $f \in C(Y)$ such that $f\left[\mathrm{Cl}_{Y} H_{1}\right] \subset\{a\}$ and $\left.\left.f\left[\mathrm{Cl}_{Y} H_{2}\right] \subset\right) b\right\}$. Let $g=f \mid X$. Then $g \in E(X, Y), g\left[H_{1}\right] \subset\{a\}$ and $g\left[H_{2}\right] \subset\{b\}$.

Assume $E(X, Y)$ is discriminating. Let $H_{1}$ and $H_{2}$ be disjoint closed subsets of $X$. If $\mathrm{Cl}_{Y} \mathrm{H}_{1} \cap \mathrm{Cl}_{Y} \mathrm{H}_{2}=\varnothing$, then there is a function $h \in C(Y)$ such that $h\left[\mathrm{Cl}_{Y} H_{1}\right] \subset\{0\}$ and $h\left[\mathrm{Cl}_{Y} H_{2}\right] \subset\{1\}$. Let $g=h \mid X$. Then $g \in E(X, Y), H_{1} \subset Z(g)$ and $H_{2} \subset Z(g-1)$. Therefore $Y \leqslant w(Z(E(X, Y)))$.

If $\mathrm{Cl}_{w(Z[E(X, Y)])} H_{1} \cap \mathrm{Cl}_{w(Z[E(X, Y)])} H_{2}=\varnothing$ then there are $F_{1}, F_{2} \in Z(E(X, Y))$ such that $H_{1} \subset F_{1}, H_{2} \subset F_{2}$ and $F_{1} \cap F_{2}=\varnothing$. Since $E(X, Y)$ is discriminating there is a function $g \in E(X, Y)$ such that $g\left[F_{1}\right] \subset\{0\}$ and $g\left[F_{2}\right] \subset\{1\}$. There is a function $h \in C(X)$ such that $h \mid X=g$. Then $h\left[F_{1}\right] \subset\{0\}$ and $h\left[F_{2}\right] \subset\{1\}$. Therefore

$$
\mathrm{Cl}_{Y} F_{1} \cap \mathrm{Cl}_{Y} F_{2}=\varnothing \text { and } w(Z(E(X, Y))) \leqslant Y \text {. }
$$

\subsection{Corollary. $C^{*}(X)$ is discriminating.}

Proof. $C^{*}(X)=E(X, \beta X)$ and $\beta X=w(Z(X))$.

2.14. Theorem. If $A \subset C(X)$ and $S \subset X$, then $\{f \mid S: f \in A\}$ is discriminating if and only if $A$ discriminates $\{S \cap H: H \in Z(A))\}$-sets. 
Proof. Let $\{f \mid S: f \in A\}$ be discriminating. Let $H_{1}$ and $H_{2} \in Z[A]$ such that $S \cap H_{1} \cap H_{2}=\varnothing$, and let $a, b \in R . S \cap H_{1}$ and $S \cap H_{2}$ are disjoint zero sets of $\{f \mid S: f \in A\}$, so there is a function $g \in A$ such that $(g \mid S)\left[H_{1} \cap S\right] \subset\{a\}$ and $(g \mid S)\left[H_{2} \cap S\right] \subset\{b\}$ so $A$ discriminates $\{S \cap H: H \in Z[A]\}$-sets.

Let $A$ discriminate $\{S \cap H: H \in Z[A]\}$-sets. Let $F_{1}$ and $F_{2}$ be disjoint zero sets of $\{f \mid S: f \in A\}$ and let $a, b \in R$. There are zero sets $H_{1}$ and $H_{2} \in Z(A)$ such that $F_{1}=H_{1} \cap S$ and $F_{2}=H_{2} \cap S$ and there is a function $f \in A$ such that $f\left[H_{1} \cap S\right] \subset\{a\}$ and $f\left[H_{2} \cap S\right] \subset\{b\}$. Therefore $(f \mid S)\left[F_{1}\right] \subset\{a\}$ and $(f \mid S)\left[F_{2}\right] \subset\{b\}$ so $\{f \mid S: f \in A\}$ is discriminating.

Since "discriminating" is a generalization of the "completely separated" concept from Urysohn's Extension Theorem and $Z(A)$-embedding is a generalization of $C^{*}$-embedding, it is logical that there be some relationship between the two concepts. In the following theorems we investigate this relationship.

2.15. THEOREM. Let $A \subset C(X)$ be discriminating, let $S \subset X$ and let $S$ be $Z(A)$ embedded in $X$, then $\{f \mid S: f \in A\}$ is discriminating.

Proof. Let $F_{1}$ and $F_{2}$ be disjoint zero sets of $\{f \mid S: f \in A\}$ and let $a, b \in R$. Then there are functions $g_{1}$, and $g_{2} \in A$ such that $F_{1}=Z\left(g_{1}\right) \cap S$ and $F_{2}=Z\left(g_{2}\right) \cap S$. Since $S$ is $Z[A]$-embedded in $X$, there are functions $f_{1}$ and $f_{2} \in A$ such that $F_{1}=Z\left(f_{1}\right) \cap S, F_{2}=Z\left(f_{2}\right) \cap S$ and $Z\left(f_{1}\right) \cap Z\left(f_{2}\right)=\varnothing$. Since $A$ is discriminating, there is a function $h \in A$ such that $g\left[Z\left(f_{1}\right)\right] \subset\{a\}$ and $h\left(Z\left(f_{2}\right)\right] \subset\{b\}$. Therefore $(h \mid S)\left[F_{1}\right] \subset\{a\}$ and $(h \mid S)\left[F_{2}\right] \subset\{b\}$. Hence $\{f \mid S: f \in A\}$ is discriminating.

2.16. Theorem. Let $A$ be a subring of $C(X)$ which contains a non-zero constant function $a$ and let $S \subset X$ be such that $\{f \mid S: f \in A\}$ is discriminating, then $S$ is $Z[A]-$ embedded in $X$.

Proor. Let $f_{1}$ and $f_{2} \in A$ such that $Z\left(f_{1}\right) \cap Z\left(f_{2}\right) \cap S$ is empty. Then there is a function $g \in A$ such that $(g \mid S)\left[Z\left(f_{1}\right) \cap S\right] \subset\{0\}$ and $(g \mid S)\left[Z\left(f_{2}\right) \cap S\right] \subset\{a\}$. Let $h=g-a$, then $h \in A, Z\left(f_{1}\right) \cap S \subset Z(g), Z\left(f_{2}\right) \cap S \subset Z(h)$ and $Z(g) \cap Z(h)=\varnothing$. Therefore $S$ is $Z[A]$-embedded in $X$.

2.17. Corollary. Let $A$ be a subring of $C(X)$ such that $A$ is discriminating and $A$ contains a nonzero constant function. If $S \subset X$, then $X$ is $Z(A)$-embedded in $X$ if and only if $\{f \mid S: f \in A\}$ is discriminating.

A closed sublattice of $E(X, w(F))$ which discriminates $F$-sets actually equals $E(X, w(F))$. To prove this we will use the following lemma as stated by Simmons (1963), p. 158.

2.18. Lemma. Let $X$ be a compact space, and let $L$ be a closed sublattice of $C(X)$ with the following property: if $x$ and $y$ are distinct points of $X$ and $a$ and $b$ are any 
two real numbers, then there exists a function $f$ in $L$ such that $f(x)=a$ and $f(y)=b$. Then $L=C(X)$.

2.19. THEOREM. If $F$ is a normal base on $X$ and $L$ is a closed sublattice of $E(X, w(F))$ such that $L$ discriminates $F$-sets, then $L=E(X, w(F))$.

Proof. Let $H=\{f \in C(w(F)): f \mid X \in L\}$.

(1) $H$ is closed. Let $f_{n} \in H$, and $g=\lim _{n} f_{n}$. Then

$$
g \in C(w(F)) \text { and } g \mid X=\lim _{n}\left(f_{n} \mid X\right) \in L .
$$

(2) $H$ is a sublattice of $C(w(F))$. Let $f, g \in H$. $(f \vee g) \mid X=(f \mid X) \vee(g \mid X) \in L$ and $(f \wedge g) \mid X=(f \mid X) \wedge(g \mid X) \in L$. Therefore $f \vee g$ and $f \wedge g \in H$.

(3) If $x, y \in w(F), x \neq y$ and $a, b \in R$, then there is a function $f \in H$ such that $f(x)=a$ and $f(y)=b$. There exist $F_{1}$ and $F_{2} \in F$ such that $x \in \mathrm{Cl}_{w(F)} F_{1}, y \in \mathrm{Cl}_{w(F)} F_{2}$ and $F_{1} \cap F_{2}=\varnothing$. Then there exists $g \in L$ such that $g\left[F_{1}\right] \subset\{a\}$ and $g\left[F_{2}\right] \subset\{b\}$. $L \subset E(X, w(F))$ so there is a function $f$ in $C(w(F))$ such that $g=f \mid X$. Then $f(x) \in \mathrm{Cl}_{R} f\left[F_{1}\right]=\mathrm{Cl}_{R} g\left[F_{1}\right] \subset\{a\}$ and $f(y) \in \mathrm{Cl}_{R} f\left[F_{2}\right] \subset\{b\}$.

Therefore by the previous lemma $H=C(w(F))$. If $f \in E(X, w(F))$, then there is a function $g$ in $C(w(F))$ such that $g \mid X=f$. $g \in H$ so $f \in L$. Therefore $L=E(X, w(F))$.

Simmons (1963), p. 159 also has a proof of the lemma which states:

2.20. Lemma. Every closed subring of $C(X)$ is a closed sublattice.

Therefore Theorem 2.19 could also have been stated as follows:

2.21. TheOREM. Let $F$ be a normal base on $X$. Let $A$ be a closed subring of $E(X, w(F))$ which discriminates $F$-sets, then $A=E(X, w(F))$.

Conversely, if $A=E(X, w(F))$, then $A$ discriminates $F$-sets.

2.22. THEOREM. Let $F$ be a normal base on $X$, then $E(X, w(F))$ discriminates F-sets.

Proof. Let $F_{1}, F_{2} \in F$ such that $F_{1} \cap F_{2}=\varnothing$ and let $a, b \in R . \mathrm{Cl}_{w(F)} F_{1}$ and $\mathrm{Cl}_{w(F)} F_{2}$ are disjoint closed subsets of the normal space $w(F)$; so by Urysohn's Lemma there is a function $h \in C(w(F))$ such that

$$
h\left[\mathrm{Cl}_{w(F)} F_{1}\right] \subset\{a\} \text { and } h\left[\mathrm{Cl}_{w(F)} F_{2}\right] \subset\{b\} .
$$

If $g=h \mid X$, then $g \in E(X, w(F)), g\left[F_{1}\right] \subset\{a\}$ and $g\left[F_{2}\right] \subset\{b\}$. Therefore $E(X, w(F))$ discriminates $F$-sets.

Combining the results of previous theorems we obtain the following necessary and sufficient conditions for a subset of $E(X, w(F))$ to be all of $E(X, w(F))$. 
2.23. TheOREM. Let $F$ be a normal base on $X$. Let $L \subset E(X, w(F))$. Then $L=E(X, w(F))$ if and only if

(1) $L$ is closed in $C(X)$;

(2) $L$ is a sublattice of $C(X)$; and

(3) L discriminates $F$-sets.

Proof. If $L=E(X, w(F)), L$ is closed since $C(w(F))$ is closed. $L$ is a sublattice of $C(X)$ by Theorem 2.7. $L$ discriminates $F$-sets by Theorem 2.22 .

If $L$ satisfies the three conditions then $L=E(X, w(F))$ by Theorem 2.19.

By Lemma 2.20, $L$ is a closed sublattice of $C(X)$ if and only if $L$ is a closed subring of $C(X)$. Therefore Theorem 2.23 could also have been stated as follows.

2.24. TheOREM. Let $F$ be a normal base on $X$. Let $A \subset E(X, w(F))$, then $A=E(X, w(F))$ if and only if

(1) $A$ is closed in $C(X)$;

(2) $A$ is a subring of $C(X)$; and

(3) A discriminates F-sets.

By Theorem 2.11 we know that an inverse closed Wallman ring $A$ which contains all the real constant functions discriminates $Z(A)$-sets. Therefore as a corollary to Theorem 2.24 we have the following.

2.25. TheOREM. Let $A$ be a Wallman ring on $X$ such that $A \subset E(X, w(Z(A)))$. If $A$ is uniformly closed, and inverse closed then $A=E(X, w(Z(A)))$.

Proof. As was noted in Bentley and Taylor (1975), Corollary 3.4, an inverse closed Wallman ring contains all the rationals. Therefore a Wallman ring which is both inverse closed and uniformly closed contains all the real constants.

The next theorem generalizes the Stone-Weierstrass Theorem so we call it the Stone-Weierstrass Theorem for Wallman lattices.

2.26. THEOREM. Let $A$ be a subset of $C(X)$ such that $Z[A]$ is a normal base on $X$ and $A \subset E(X, w(Z(A)))$. Let $L$ be a sublattice of $C(X)$ such that $L$ is closed in $A$ and $L$ discriminates $Z[A]$-sets. Then $L=A$.

Proof. Let $H=\mathrm{Cl}_{Z(X, w(Z(A)))} L . L \subset H$ and $H$ is a closed sublattice of $E(\mathrm{X}, w(Z(A)))$. Since $L$ discriminates $Z[A]$-sets, $H$ discriminates $Z(A)$-sets. Therefore $H=E(X, w(Z(A)))$. $L$ is closed in $A$ so $H \cap A=L$. Also $A \subset H$, so $H \cap A=A$. Therefore $L=A$.

Similarly we have the Stone-Weierstrass Theorem for Wallman rings. 
2.27. THEOREM. Let $A$ be a sublattice of $C(X)$ such that $Z(A)$ is a normal base on $X$ and $A \subset E(X, w(Z(A)))$. Let $L$ be closed in $A$, let $L$ be a subring of $C(X)$ which contains the real constants, and let $L$ discriminate $Z(A)$-sets. Then $L=A$.

Proof. The hypotheses of Theorem 2.27 include all the hypotheses of Theorem 2.26 except $L$ being a sublattice of $C(X)$. To show $L$ is a sublattice of $C(X)$, it suffices to show $|f| \in L$ for each $f \in L$.

Let $t=\sup \{|f(x)|: x \in X\}$ and let $\varepsilon>0$. There is a polynomial $p:[-t, t] \rightarrow R$ such that $p$ has real coefficients and ||$r|-p(r)|<\varepsilon$ for all $r \in[-t, t]$ (Weierstrass Approximation Theorem). Then ||$f|(x)-p(f(x))|=|| f(x)|-p(f(x))|<\varepsilon$ for all $x \in X . p \circ f \in L$ and $|f| \in A$ so $|f| \in \mathrm{Cl}_{A} L=L . L$ is a sublattice of $C(X)$.

If we let $A \subset C^{*}(X)$ be an algebra on $X$ we find $A=E(X, w(Z[A]))$ and also obtain some interesting results involving $(B, A)$-embedding.

2.28. Defintion (Hager, 1969). $A$ is an algebra on $X$ if and only if:

(1) $A$ is a subring of $C(X)$;

(2) $A$ contains all real valued constant functions;

(3) $A$ separates points and closed sets;

(4) $A$ is closed under uniform convergence; and

(5) $A$ is inverse closed.

We will show that every algebra on $X$ is a Wallman subring of $C(X)$. Lemma 2.20 established that every closed subring of $C(X)$ is a closed sublattice of $C(X)$ and so we have the following result which was observed by Mrówka (1964).

2.29. THEOREM. If $A$ is an algebra on $X$, then $A$ is a lattice.

Biles (1970) established the following.

2.30. THEOREM. Let $A$ be a subring of $C(X)$ such that $Z[A]$ is a base for the closed sets of $X$ and if $f \in A$, then $|f| \in A$. Then $A$ is a Wallman ring on $X$.

If $A$ is a lattice and $f \in A$, then $|f| \in A$. So $|f| \in A$ for each $f$ in an algebra $A$. Therefore we have proven that every algebra on $X$ is a Wallman ring on $X$.

\subsection{THEOREM. Every algebra on $X$ is a Wallman subring of $C(X)$.}

In fact, if $A \subset C^{*}(X)$ is an algebra on $X$, then $A$ is the Wallman ring $E(X, w(Z(A)))$. To prove this we will use the following theorem which is due to Isbell (1958).

2.32. Theorem. If $A$ is an algebra on $X$, then $A$ is closed under composition.

2.33. TheOREM. If $A \subset C^{*}(X)$ is an algebra on $X$, then $A=E(X, w(Z(A)))$. 
Proof. $A$ is a Wallman ring which is closed under composition so by Theorem 2.3, $A \subset E(X, w(Z(A)))$. By Theorem 2.11, $A$ discriminates $Z(A)$-sets. Therefore the hypotheses of Theorem 2.24 are satisfied and $A=E(X, w(Z(A)))$.

From this we are able to show that if $A$ is an algebra of bounded functions on $X$ and $B$ is an algebra of bounded functions on $S$, where $S \subset X$, then $S$ is $(B, A)$ embedded in $X$ if and only if $B=\{f \mid S: f \in A\}$.

2.34. TheOREM. Let $A$ be an algebra on $X$ such that $A \subset C^{*}(X)$. Let $S \subset X$. Let $B$ be an algebra on $S$ such that $B \subset C^{*}(S)$. Then $S$ is $(B, A)$-embedded in $X$ if and only if $B=\{f \mid S: f \in A\}$.

Proof. $A=E(X, w(Z(A)))$ and $B:=E(S, w(Z(B)))$. Let $S$ be $(B, A)$-embedded in $X$. If $f \in A$, then there is a function $g \in C(w(Z(A)))$ such that $f=g \mid X$. If $h^{\prime}=g \mid C l_{w(Z(A))} S$, then since $\mathrm{Cl}_{w(Z(A))} S \cong w(Z(B))$ there is a function $h \in C(w(Z(B)))$ such that $h\left|S=h^{\prime}\right| S=f \mid S$. Therefore $f \mid S \in B$ and $\{f \mid S: f \in A\} \subset B$.

If $f \in B$, then there is a function $g \in C(w(Z(B)))$ such that $f=g \mid S$, and consequently a function $h \in C\left(\mathrm{Cl}_{w(Z(A))} S\right)$ such that $h \mid S=f$. Since $\mathrm{Cl}_{w(Z(A))} S$ is compact, it is $C^{*}$-embedded in $w(Z(A))$ and $h$ has a continuous extension $h^{\prime}$ to $w(Z(A))$. Then $h^{\prime} \mid X \in A$, and $\left(h^{\prime} \mid X\right) \mid S=f$, so $B \subset\{f \mid S: f \in A\}$.

Conversely, if $B=\{f \mid S: f \in A\}$, then by Theorem 2.39 of Bentley and Taylor (1978), $S$ is $Z(A)$-embedded in $X$ and by Theorem 2.40 of Bentley and Taylor (1978), $S$ is $(B, A)$-embedded in $X$.

The next two theorems are corollaries to this theorem.

2.35. ThEOREM. If $A$ is a sublattice of $C(X), Z(A)$ is a normal base on $X, A$ is discriminating, $A \subset E(X, w(Z(A))), S \subset X, S$ is $Z(A)$-embedded in $X, B \subset C(S), Z(B)$ is a normal base on $S, B \subset E(S, w(Z[B]))$ and $\{f \mid S: f \in A\}$ is closed in $B$, then $S$ is $(B, A)$-embedded in $X$ if and only if $B=\{f \mid S: f \in A\}$.

Proof. Let $L=\{f \mid S: f \in A\}$. $L$ is a sublattice of $C(S)$ and $L$ is closed in $B$. Since $S$ is $Z(A)$-embedded in $X, A \cong{ }_{S} L$, by Corollary 2.37 of Bentley and Taylor (1978). If $S$ is $(B, A)$-embedded in $X$, then by Theorem 2.23 of Bentley and Taylor (1978), $A \cong S B$. Therefore $L \cong B$. Since $A$ is discriminating and $S$ is $Z(A)$-embedded in $X$, $L$ is discriminating. Therefore $L$ discriminates $Z(B)$-sets. Now we have satisfied the hypotheses of Theorem 2.26 so $L=B$.

Conversely, if $L=B$, then since $\{f \mid S: f \in A\} \cong{ }_{X} A, B \cong{ }_{S} A$. So by Theorem 2.23 of Bentley and Taylor (1978), $S$ is $(B, A)$-embedded in $X$.

2.36. ThEOREM. If $A \subset C(X), Z(A)$ is a normal base on $X, A \subset E(X, w(Z(A)))$, $S \subset X, S$ is $Z(A)$-embedded in $X, B$ is closed in $\{f \mid S: f \in A\}, B$ is a sublattice of $C(S)$ and $B$ is discriminating, then $S$ is $(B, A)$-embedded in $X$ if and only if $B=\{f \mid S: f \in A\}$. 
Proof. Let $S$ be $(B, A)$-embedded in $X$. Let $H=\{f \mid S: f \in A\}$. $Z(H)$ is a normal base on $S$, by Theorem 2.34 of Bentley and Taylor (1978). Since $S$ is $(B, A)$ embedded in $X, A \cong{ }_{S} B$. But $H \cong{ }_{S} A$ so $H \cong B$. $h \in H$ implies $h$ has an extension to a function in $A$, hence to a function in $C(w(Z(A)))$ ). But $\mathrm{Cl}_{w(Z(A)))} S \cong w(Z(H))$, so $h$ has an extension to a function in $C(w(Z(H)))$. Therefore $H \subset E(S, w(Z(H)))$. $B$ discriminates $Z(B)$-sets, consequently $Z(H)$-sets. Now by Theorem $2.26, H=B$.

If $B=\{f \mid S: f \in A\}$, then $B \cong_{S} A$. So, by Theorem 2.23 of Bentley and Taylor (1978), $S$ is $(B, A)$-embedded in $X$.

\section{REFERENCES}

All references refer to those listed at the end of the immediately preceding paper:

H. L. Bentley and B. J. Taylor (1978), "On generalizations of $C^{*}$-embedding for Wallman rings”, J. Austral. Math. Soc. 25 (Ser. A), 215-229.

The University of Toledo

Toledo

Ohio 43606, USA
IBM Corporation

Sylvania

Ohio 43560, USA 